(Aus der Königl. Universitäts-Frauenklinik zu Leipzig.)

\title{
Zur Casuistik und chirurgischen Behandlung der postoperativen allgemeinen Peritonitis.
}

\author{
Von \\ Dr. K. Hintze, Frauenarzt in Stettin. \\ (Ehemaliger Assistent der Klinik.)
}

Seitdem die Behandlung der allgemeinen eitrigen Peritonitis mehr in die Hände der Chirurgen übergegangen ist, sind zahlreiche Publicationen hierüber erschienen. Die Ansichten der verschiedenen Autoren indess über einzelne Punkte sind recht mannigfaltige und dissentirende, auch sind ihre Resultate auffallend von einander abweichende, so dass es wohl der Mühe lohnt, die Beobachtungen an neuen Fällen mitzutheilen, um an der Hand dieser und der einschlägigen Literatur eine kritische Betrachtung anzuschliessen.

Ich gebe zunächst die Beschreibung zweier Fälle, die ich während meiner Assistentenzeit in der Universitäts-Frauenklinik zu Leipzig zu beobachten Gelegenheit hatte.

1. Martha Sch., 22jährige Dienstmagd. Constitution schwächlich, Grösse $118 \mathrm{~cm}$, graciler, ausgesprochen rhachitischer Knochenbau. Lungen- und Herzbefund obne Besonderheiten. Urin eiweissfrei. I gravida mensis $\mathrm{X}$.

Am 15. 4. 97 wegen Kaiserschnittbecken (Conj. vera $6 \mathrm{~cm}$ ) in die Uniwersitäts-Frauenklinik aufgenommen.

Sectio caesarea in Aethernarkose am selben Tage Abends $6 \mathrm{Uhr}$ vom Verfasser ausgeführt. Sitz der Placenta an der vorderen Lteruswand. Stärkere Blutung aus den durchschnittenen Gefässen an der Placentarstelle, die nach Extraction des lebenden Kindes umstochen werden müssen. Im Uebrigen glatter Verlauf der Operation. Schluss der Jteruswunde durch tiefe und oberfiächliche Nähte. Bauchwunde in drei Etagen genäht. 
Schon am Abend des ersten, sehr unruhig hingebrachten Tages steigt die Temperatur auf 38,7. Der Puls ist sehr frequent, weich und klein, 124-140 in der Minute. Zunge etwas trocken, kein Erbrechen, keine Flatus. Urin spontan entleert. ' 17, 4. Die Nacht über ist Pat. sehr unruhig gewesen. Am Tage starke Leibschmerzen, viel Aufstossen, zeitweise auch Brechneigung. Urin spontan entleert, keine Flatus, Lochien blutig. Zunge trockener als gestern. Leib aufgetrieben, überall tympanitischen Schall gebend. Puls noch frequenter 148. Temp. 38,5. Gegen Nachmittag häufigeres Erbrechen, Leib noch stärker aufgetrieben, keine Flatus. Einlauf ohne Erfolg. Herzthätigkeit schwach, deshalb 2 stündlich Kampherölinjectionen. Hoher Einlauf über den Sphincter tertius hinauf auch ohne Erfolg. Pat. zeigt stark rerfallene Gesichtszüge. Puls 152. Abends 7 Uhr wird deshalb in. Aethernarkose zur nochmaligen Eröffnung der Bauchböhle geschritten. Nach Entfernung der Näbte weichen die Wundränder leicht auseinander. Die stark geblähten und injicirten Darmschlingen drängen sich danach sofort aus der Wunde heraus. Die Serosa ist überall matt, verfärbt und vielfach mit Fibrinmassen bedeckt. Zwischen den einzelnen leicht verklebten Schlingen trübe, seröse und eiterige Flüssigkeit, die in den Flanken und im kleinen Becken etwas massiger amzutreffen ist, aber im Ganzen nicht $300 \mathrm{ccm}$ übersteigt. Die Darmschlingen werden eventrirt und in trockene sterile Tücher eingehüllt. Eine Abklemmung des Darmes, die vorher nicht ausgeschlossen werden kounte, war beim Absuchen des ganzen Darmes nicht zu finden. An der Uteruswunde keine Verklebung mit dem Darm. Vorsichtiges Aus- und Abtupfen des flüssigen und plastischen Fxsudats mit trockenen Tupfern. Von dem Eiter und den Fibrinauflagerungen werden mit steriler Platinöse direct Abimpfuugen vorgenommen, deren Züchtungen Streptococcus pyogenes aureus und Bacterium coli ergeben.

Die Bauchwunde wird, nachdem die noch immer stark geblähten Därme mit vieler Mühe reponirt sind, in Etagen mittels Knopfnähte geschlossen.

Nach der Operation wird auf Application eines hohen Einlaufes mit dem zurückkommenden Wasser etwas Stuhl entleert.

In den nächsten Stunden erbricht Pat. viel. Puls klein und weich 148. Temperatur 38,6. 18. 4. Nacht unruhig. Viel Durst und Leibschmerzen. Puls noch klein und frequent 140-152. Temp. 37,2-38,0. Aufstossen noch häufiger, kein Erbrechen mehr. Abgang von Flatus. Leib noch stark aufgetrieben, Nahtstelle geröthet, aus den Stichcanälen entleert sich trübe, unangenenm riechende Flüssigkeit. Lochien reichlich, blutig gefärbt. Urin per Katheter entleert. 2stïndlich Kampherölinjectionen, mehrmals Einläufe.

20. 6. Pat. ist viel ruhiger geworden, Leib weniger empfindlich, aber noch stark aufgetrieben, eitrige Secretion aus den Stichcanälen. Zunge beginnt feucht $\mathrm{zu}$ werden. Temp. 37,2-38,4. Puls 128-142. Einlauf kommt etwas gelb gefärbt zurück. Flatus mehrmals. Aufstossen selten, kein Erbrechen. Lochien etwas eitrig. 3 mal täglich Kampherbenzoepulver. Brühe und Wein per os.

21. 4. In der Nacht $3 \mathrm{mal}$ spontan breiiger Stuhl. Pat. hat mehrere Stunden geschlafen. Aussehen bedeutend besser. Leib weniger hoch. Das untere Drittel der Bauchwunde hat sich spontan geöffnet bis auf das Peritoneum und secernirt übelriechenden Eiter, dessen bacterielle Prüfung Streptokokken und Bacterium coli ergiebt. Aus der 
Scheide massiger, übelriechender Eiterabgang. Höcbste Temp. 38,6, Puls 124. Während des Tages zahlreiche (16) diarrhöische, äusserst ühelriechende Stühle. Nahrungsaufnahme per os vermehrt. In den nächsten Tagen zunehmendes. Woblbefinden. Puls wird stetig Iangsamer, Temp. steigt nicht mehr über 38,0. Die Bauchwunde ist der Luänge nach auseinandergewichen, so dass der Serosaïberzug der mit einander verklebten Darmschlingen völlig frei liegt.

Die żunächst noch stark eiternde Wunde reinigt sich allmäblich unter 'Kampherweinumschlägen. Die 'Wundränder der' Bauchdecken werden durch Heftpflaster, an dem eine Schnürvorrichtung angebracht ist, möglichst an einander gedrängt gehalten.

Stuhlentleerungen täglich spontan.

Aus der Scheide hält die eitrige Absonderung noch bis zum 10.5. zjemlich profus an. Erst vom 14. 5. ab wird sie geringer. Am 5. 6. hat sich die Bauchwunde völlig überbäutet. Die Narbe ist ca. 2 Querfinger breit.

Am 11. 6. wird Pat. geheilt extlassen.

Der zweite Fall, den ich im Anschluss hieran mittheile, gehört streng genommen nicht in die Kategorie der postoperativen Peritonitís: $\mathrm{Da}$ aber auch hier eine durch geburtshülfliche Manipulationen von aussen zugetragene Infection der Bauchböhle vor der Operation bestand, mag dadurch die Berechtigung seiner Veroffentlichung an dieser Stelle begründet sein.

Bertha Sch., Schlossersfrau, 37 Jahre alt; wurde am 6.12. 1896 wegen Gebärunmöglichkeit in Folge eines ausgedehnten Portiocarcinoms von einem auswärtigen Arzte der Vniversitäts-Frauenklinik zngeschickt.

Es handelte sich un eine $X V$ para, die 11 mal spontan geboren und 3mal abortirt hatte. Letzte Meristruation December 1895, erste Kindesbewegungen im Juni 1896. Die Wehenthatigkeit begann am 1. 12. 1896, bald danach soll die Blase gesprungen sein. Erst am 4. 12. schickte die recht sorglose Hebamme nach ärztlicher Hülfe. Am Vormittag dieses Tages batten sich die Wehen, wie die Frau selbst berichtete, vorübergehend eingestellt, bald hatte Pat. jedoch fast unausgesetzt unter den heftigsten Schmerzen im Leibe zu klagen gehabt. Die Temperatur betrug nach ärztlicher Angabe $38,5^{\circ} \mathrm{C}$.

Da der zaerst zugerufene Arzt sich über den Fall nicht völlig klar wurde, consultirte er am Nachmittag noch einen Collegen, der uns dann aus dem oben angeführten Grunde die Pat. zuschickte.

Wir fanden bei der anämischen, collabirten, schwächlichen Frau bei einer Temperatur von 37,8 und einem kleinen frequenten Puls von .112 den Leib entsprechend dem Ende der Schwangersehaft stark vorgewölbt bis zum Rippenbogen hin. Kleine Kindstheile waren links dicht unterhalb der Rippen sehr deatlich direct durch die Bauchdecken durchzufühlen. Der Kopf war nicht auffindbar, kindliche. Herztöne konnten nicht gehört werden. Der Leib war selbst bei leisester Berührung äusserst schmerzhaft, weshalb die Untersuchung auch sehr behutsam vorgenommen werden musste.

Die Beckenmaasse waren normal. Bei der Exploration konnte man einen im Douglas liegenden ca. kindskopfgrossen; prallelastischen Tumor fühlen, der den Beckeneingang völlig ausfüllte. Die Portio war kurz 
und schlaff, wie im Puerperium; sie stand dicht hinter der Symphyse und etwa in der Höhe ihres unteren Randes. Der Muttermund war quergespalten und für drei Finger bequem durchgängig. Das Uteruscavum wurde, soweit man es abtasten konnte, leer befunden. Aus den Genitalien entleerte sich eine mässige Menge blutiger Flüssigkeit.

Da nach diesen Untersuchungsbefund jedenfalls die Frucht frei in der Bauchhöhle lag, blieb nichts anderes zur Rettung der Frau übrig, als die Laparotomie.

Nach eingeleiteter Aethernarkose liess sich nun der etwa in Nabelhöhe stehende Uterus völlig von dem in der freien Bauchhöhle liegenden Kind abgrenzen, was vorher wegen des äusserst schmerzhaften Leibes nicht möglich gewesen war. Der im Douglas liegende Tumor. wurde als ein Ovarialcystom angesprochen. Bei einer Austastung des Uterus fand man denselben wie auch vorher gleichfalls, völlig leer. Eine Rissstelle war aber nicht zu palpiren. Obwohl alles für eine Ruptur sprach, wurde die Eventualität einer geplatzten Extrauteringraviditat nicht ganz ausser Acht gelassen.

Bei der nun sofort von Herrn Geheimrath $Z$ w eifel vorgenommenen Laparotomie entleerte sich aus der geöfneten Bauchhöhle fast ein Liter theils geronneres, theils flüssiges Blut. Das mittelgrosse ausgetragene, todte Kind befand sich im Zusammenhang mit der Placenta im freien Bauchraum zwischen den Darmschlingen. Placenta und Kind zeigten bereits beginnende Fäulnisserscheinungén und verbreiteten einen sehr hässlichen Fötor, 'dem entsprechend zeigten auch die Darmschlingen vielfach schmutzig graue, übelriechende frische Bèläge.

Nachdem das Kind mit seinen Anhängen sehnell entfernt und die Bauchhöhle vom Blut gesäubert war, wurde, sofort nach der Quelle der Blutung gesucht, es konnte eine solche jedoch nicht gefunden werden. Der im Douglas liegende Ovarialtumor wurde nun heranisgewälat und nachdem der Stiel durch. Partieenligaturen versorgt war, nit dem $\mathrm{Pa}$ quelín abgetragen. Er erwies sich als ein Dermoid. Da an beiden Admexen eine' Eiinsertion micht $\mathrm{zu}$ finden war, auch nicht in der freien Bauchhöhle, so wurde nochmals der Uterus auf eine Ruptur abgesucht. Erst nachdem von der Scheide aus mit zwei Fingern eingegangen war, konnte man nun durch die combinirte Betastung an der vorderen Cervixwand dicht hinter der Blase eine schräg verlaufende, jetzt $5 \mathrm{~cm}$ lange Rissstelle constatiren. Dieselbe blutete absolut nicht.

Wegen der bereits bestehenden Infection der Bauchhöhle wurde von einer Naht dieses Risses Abstand genommen, derselbe vielmehr nur nach der Vagina zu mit Jodoformgaze tamponirt.

Nach ausgiebiger Toilette der Bauchhöhle mit trockenen Tupfern Schluss der Bauchdecken in drei Etagen.

Der Leib blieb zunächst noch stark aufgetrieben und ziemlich sehmerzhaft.

Am 1. Tage p. op. Flatus, Urin spontan, kein Erbrechen.

Am 2. Tage nach reichlichem Flatusabgang Allgemeinbefinden besser. Es machte sich bei der ziemlich collabirt aussehenden Kranken ein überaus penetranter süsslicher Foetor ex ore bemerkbar, der erst etwas wich, als am 4. und 5 . Tage reichliche, übelriechende diarrhoische Stühle entleert wurden.

Der Puls war zu Anfang sehr frequent und klein - deshalb Kampherölinjectionen mehrmals täglich - die Temperatur schwankte zunächst innerhalb normaler Grenzen. Am 6. Tage wurde der Krank- 
heitsverlauf noch durch eine Pneumonie mit Temperatursteigerungen über $39,0^{\circ}$ complicirt.

$\mathrm{Zu}$ gleicher Zeit konnte auch ein sich innerhalb der Bauchwunde bildender Bauchdeckenabscess constatirt werden, aus dem sich nach Entfernung einiger Nähte unter Auseinanderweichen der Wundränder ein stark übelriechender Eiter entleerte, aus dem das Becterium coli gezüchtet werden konnte, das sich auch aus dem während der Operation mit einem sterilen Tupfer entfernten plastischen Secret hatte züchten lassen.

In der Folgezeit gingen die Erscheinungen von Seiten der Lunge allmälig zurück, auch die Bauchdecken vereinigten sich bald, so dass Patientin am 26. Tage aufstehen und am 36. geheilt entlassen werden konnte.

Ventiliren wir nun hiernach zunächst die Frage: wann soli man operiren?

Wer an einem zahlreichen chirurgischen Material Gelegenheit gehabt hat, häufiger primäre oder post operationem auftretende Peritonitiden und Ileusfälle - denn nicht immer ist zwischen beiden eine differentielle Diagnose möglich - zu beobachten, der weiss, wie schwer es oft ist, den richtigen Zeitpunkt zum chirurgischen Eingriff zu treffen: Magenausspülungen, hohe Einläufe, subcutane oder intravenöse Kochsalzinfusionen Michaux (1), Monod(2) im Vereine mit Excitantien werden mit Gespanntheit auf ihre Wirkung applicirt. Eine wenn auch geringe Besserung berechtigt vielleicht zo guter Hoffnung.

Unser zweiter Fall ist bezüglich des Entschlusses zur Operation nicht heranzuziehen, weil hier die Indication in der Ruptura uteri bestand.

Im ersten Falle wurde nach kaum 2 mal 24 Stunden p. op. zur nochmaligen Oeffnung der Bauchhöhle gesehritten. Wir liessen uns dazu bewegen, weil bei deutlichen Zeichen einer allgemeinen Peritonitis die Patientin zusehends verfiel, auch ein Ileus nicht auszuschliessen war. Dass man wegen des collabirten Aussehens vor einem operativen Eingriff nicht zurückzuschrecken braucht, beweisen mit unseren Fällen noch zahlreiche andere.

Körte (3 und 4) erwähnt noch besonders, dass viele seiner Kranken in sehr geschwächtem Zustande zur Operation kamen, mehrere sogar in so verzweifeltem, dass er auch bei thunlichster Vereinfachung des Eingriffs ein Ueberstehen nicht erwarten konnte und trotzdem genas auch ron diesen letzteren einer. Wo allerdings bereits schnappende, oberflächliche Athmung und Cyanose der Extremitäten, auch wenn der Puls noch fühlbar ist, zu constatiren ist, räth auch Körte von einer Operation Abstand zu nehmen. 
Fast überall in der Literatur finden wir ein Eintreten für eine möglichst frühzeitige Operation (A b bé(5), v. Erlach (6), Oberst(7), Lawson Tait (8), Körte u. a.); jedenfalls soll, sobald ein Exsudat nachweisbar ist, zur Operation geschritten werden.

Mit Recht sagt von Winckel (9): wenn in irgend einem Organ, einer Drüse, einem Muskel ete. eine Eiterung besteht und die ursprüngliche Incision. sich geschlossen oder eine Senkung sich gebildet hat, so zögert man keinen Angenblick, eine neue Incision zu machen und den Eiter zu entleeren; warum soll man beim Peritoneum anders verfahren?

Sippel (10) bält es für falsch, vornehmlich bei den nach gynäkologischen Operationen im kleinen Becken auftretenden Peritonitiden, wenn man so lange warten wollte, bis erst ein palpatorisch nachweisbares Exsudat eingetreten ist.

In unserem ersten Falle konnten wir vorher ein Exsudat nicht nachweisen, weil es die stark geblähten Darmschlingen überlagerte und auch relativ gering war.

Nach den einzelnen Beriehten aus der Literatur ist kaum ein Fall zur Genesung gekommen, wo später als am 4. Tage nach dem Beginn der Peritonitis operirt wurde.

Bezüglich der Bezeichnung: allgemeine Peritonitis herrschen in der Literatur einige Meinungsverschiedenheiten.

Wenn wir Körte's $(3,4)$ glänzende Resultate mit denen anderer Autoren, wie Senn (11), Faure (12), Forgue (13) vergleichen, so liegt es nahe, daran zu denken, dass Körte für die Bezeichnung der allgemeinen Peritonitis vielleicht andere Gerichtspunkte gewählt hat, als wir sie bei den Autoren mit viel ungünstigeren Resultaten finden. Und in der That fasst Körte $(3,4)$ Senn (11) gegenüber die Bezeichnung: allgemeine Peritonitis nicht so auf, dass nun auch das totale Peritoneum parietale und viscerale erkrankt sein muss. Es kann wohl sein, dass hier und dort ein Theil der Peritonealhöhle auf kleine Strecken hin intact gewesen sein mag, wenn nur der grössto Theil des Peritoneums oder die Bauchhöhle, soweit sie dem Auge zugänglich war, erkrankt gewesen ist. Denn klinisch, sagt Körte (3), macht eine solche allgemeine Peritonitis dieselben Symptome wie eine totale diffuse.

König (4) steht nicht ganz auf dem Boden der Körte'schen Erörterungen über die allgemeine Peritonitis. Es sind ihm Fälle bekannt, wo er den ganzen Bauch voller eitriger Exsudatmassen gefunden hat; dazwischen haben aber doch immer Abkapselungen 
bestanden und Partieen, die frei von Entzündung. und Eiterung waren.

Deanesley (14) rechnet zur allgemeinen Peritonitis alle Fälle, wo sich Exsudat im kleinen Becken; den beiden Flanken und zwischen den Därmen vorfindet.

Lennander(4) betont, dass die Ausdrücke diffuse und allgemeine Peritonitis sehr missbraucht werden. Nach ihm muss zwischen einer Peritonitis oberhalb. und unterhalb des Colon transyersum unterschieden werden; bei dieser letzteren wieder eine Peritonitis zwischen den Dünndarmschlingen von einer solehen in der Peripherie des Bauches getrennt werden, die prognostisch weit günstiger ist wegen der viel kleineren Resorptionsfläche.

Körte (3) unterscheidet im allgemeinen drei Arten von allgemeiner Peritonitis:

1. die septische, ohne wesentliche Exsudatbildung,

2. die jauchig eiterige ohne Verklebungen und

3. die jauchig eiterige mit Verklebungen.

Von diesen eignet sich die erste Form nicht zur Operation, weil hier die allgemeinen septischen Erseheinungen in den Vordergrund treten.

Am meisten 'Erfolg nach Operation versprechen die unter 3 angeführten Formen.

In seiner späteren Abhandlung (4) aus dem Jahre 1897 spricht er nư yon 2 Hauptgruppen und zählt zu den ersten die peritoneale Sepsis, zur zweiten alle mit einem Exsudat einhergehenden Peritonitiden.

Wenden wir uns nun zur Frage der Technik bei der Operation, so stimmen fast alle Autoren, so verschieden auch die Ansichten hinsichtlich anderer Punkte sind, in drei Punkten ziemlich allgemein überein.

Das sind: breite Eröffnung der Bauchhöhle, Entleerung des Exsudats und Drainage.

Was die Eröffnung der Bauchhöhle anlangt, so wird es bei vorhergegangener Laparotomie fast immer genügen, die frisch verklebte Wunde durch Entfernung der Fäden wieder zu öffnen.

Jedenfalls wird im Allgemeinen die Eröffnung in der Mittellinie der besseren. Uebersicht wegen bevorzugt. Wo mit Sicherheit das Exsudat in der seitlichen Unterbauchgegend diagnosticirt ist, soll man nach Sonnenburg (4) hier zunächst incidiren, weil man bei nicht ganz sicherer Diagnose auf diffuse. Peritonitis sich 
mit dem Aufsuchen des kranken Herdes begnügen und die:Gefahr der Eröffnung des vielleicht noch wenig veränderten Bauchraumes vermeiden kann. Der Medianschnitt hat Sonnenburg bei diffuser Peritonitis niemals : Erfolg gebracht.

Bei der Entfernung des Sekrets finden wir verschiedene Verfahren. Während viele sich auf vorsichtiges Austupfen des Exsudats beschränken, wobei sie die Adhäsionen durchaus geschont haben wollen, und durch Lagerung auf die Seite den Abfluss des Eiters unterstützen, Deanesly (14), Schloffer (15), Vallas (16), Berard(17), empfehlen andere möglichst ausgiebige Bespülungen der Peritonealflächen mit temperirten Flüssigkeiten bis zu 40 Litern, Bode (18), Sykow (19), Gould (20), Picherin et Petit (21). Meist wird dazu physiologische Kochsalzlösung bevorzugt, einige nehmen nur steriles Wasser. Maxwell (22) empfiehlt sogar wie auch Bode (23) die Spülungen noch nach der Operation durch die Drains fortzusetzen. Körte spült nur bei Vorhandensein von Adhäsionen, um dieselben nicht zu lösen. Er hält, wie auch Oberst (7), Berard (17) eine Desinfection der Bauchhöhle für unmöglich; sie soll deshalb auch nicht angestrebt werden. Berard (17) betont ausserdem auch die schädliche Rückwirkung der Spülungen auf's Herz, ebenso macht. Floderus (24) darauf aufmerksam. von Winckel (9a) nennt die Zahl der Operateure, welche die Spülungen verwerfen, eine verhältnissmässig geringe, als hauptsächlichsten Gegner führt er' Reichel an, nächst ihm Pölehen, Witzel, Gargitano und Sänger. Winckel selbst empfiehlt in seinem oben citirtén Aufsatze (9a) die Auswaschungen der Bauchböhle bei postoperativer Peritonitis insonderheit wegen der vielen günstigen Erfolge, wèlche die einzelnen Autoren dadurch erzielt haben. Von Anhängern der Spülungen erwähnt er namentlich fünfzehn. Um so auffälliger ist nach diesen Auseinandersetzungen unter den Schlusssätzen seiner Arbeit der Passus: „Ausspülungen der Bauchhöhle unterbleiben."

In unsern beiden Fällen wurde sowohl das plastische wie eiterige Exsudat mit trockenen Tupfern so ausgiebig wie möglich entfernt. Dabei die tiefliegendsten Theile der Bauchhöhle, das kleine Becken, der Raum unter Leber and Milz wir operirten nicht in Beckenhochlagerung - besonders beachtet.

Leichte Verklebungen der Darmschlingen fanden. wir nur in dem ersten Falle, hier haben wị sie beim Absuchen des Darms 
trennen müssen, um uns Klarheit über den vermutheten Ileus zu verschaffen.

Bode (18) löst bei seinen ausgiebigen Spülungen die unter einander verklebten Darmschlingen, während die Mehrzahl gerade das Schonen der Adbäsionen betont.

Um möglichst schonend vorzugehen, vermeiden viele auch die Eventration, Senn (11) warnt sogar direct davor.

Wir haben in beiden Fällen eventrirt, einmal des nicht völlig: auszuschliessenden Ileus wegen, das andere Mal, um eine bessere Orientirung über die schwer aufzufindende Rupturstelle am Uterus zu gewinnen.

Finney (25) eventrirt methodisch bei Peritonitis und packt die Darmschlingen in heisse mit Kochsalzlösung getränkte Compressen ein, wie er auch mit derartigen Tüchern die ganze Bauchhöhle energisch austupft.

Bei stark geblähtem Darm empfiehlt Mac Cosh (26), da bei ihm die Herstellung der Peristaltik eine grosse Hauptrolle spielt, Darmincisionen zur Entleerung des Kothes. Nach der Incision injicirt er ausserdem noch in eine hohe Darmschlinge 1-2 Unzen einer concentrirten Magnesiasulfatlösung. Er will dadureh bedeutend bessere Resultate erzielt haben als früher ohne diese Maassregel. Von 8 Fällen hat er auf diese Weise 6 retten können, während er vordem unter 5347 verloren hat. Ob sein Vorschlag viele Nachahmer finden wird, möchte ich bezweifeln. Es ist überhaupt anzustreben, den Eingriff möglichst einfach und kurz zu gestalten. Auf die Entlastung der Bauchhöhle durch die Entfernung des Eiters ist zunächst der Hauptwerth zu legen. Etwaige noch anzuschliessende Operationen verspare man sich lieber auf spätere Zeiten, falls sie nicht absolut dringend sind. Später operirt man dann anter viel günstigeren Verbältnissen, ja man wird vielleieht von dem geplạnten Eingriff auch gänzlich Abstand nehmen können.

An die Frage der Entfernung der Exsudatmassen schliesst sich eng die der Drainage an. Hier herrseht bei fast allen Autoren die gleiche Anschauung der Nothwendigkeit einer solehen.

Wenn sich auch viele Autoren wohl bewusst sind, dass die Ableitung der Sekrete aus der Bauchhöhle nur in sehr unvollkommener Weise erfolgen könne wegen der ungünstigen anatomischen Verhältnisse, so halten sie es doch für angezeigt für Abfluss der Sekrete wenigstens die Möglichkeit zu geben. Am meisten werden dazu mit Jodoformgaze unwickelte Gummidrains empfohlen. 
Die Drainage wird sich nach dem jedesmaligen Befunde richtẹn und besonders die gefährdetsten Stellen der Bauchhöhle, wo das Sekret sich zu stauen droht, beachten müssen. Für die nach gynäkologischen Operationen auftretenden Peritonitiden, wo sich das Sekret vornehmlich im kleinen Becken ansammelt, wird eine Drainage nach der Scheide zu von besonderem Vortheil sein.

Wir haben in keinem unserer Fälle drainirt. Bei der Ruptura uteri ist nach der Scheide zu allerdings der Riss lose tamponirt, was ja einer Drainage nahe kommt.

Bei der Sectio eaesarea hatten wir eine sichere Quelle, von der die Infection ausgegangen zu sei schien, bei der Operation nicht finden können. Jedenfalls war auch das Uterusinnere infieirt, dafür spricht die reichliche Eiterabsonderung mit den Lochien. Für den Uterus war ja durch die anatomischen Verhältnisse eine Drainage von selbst geschaffen. Wenn auch der Fall günstig verlaufen ist, so können wir doch nicht leugnen, dass nach der ganzen Sachlage auch hier eine Drainage vom Douglas aus nach der Scheide am Platze gewesen wäre.

Wir nahmen eine solche nicht mehr vor, weil wir bei der Aussichtlosigkeit des Falles die Operation möglichst schnell beenden wollten.

Anstatt der Drainage lassen einige Operateure bisweilen die Bauchwunde offen, so König (4) und Gluck (27). Auch James Israel (4) empfiehlt in verzweifelten Fällen davon Gebrauch zu machen, um den intraabdominellen Druck auf ein Minimum herabzusetzen. Die mangelnde vordere Bauchwand ersetzt er durch eine unter die Bauchwandränder geschobene grosse Jodoformgazeschürze.

Während die meisten Operateure ausser den bereits oben erwähnten Gesichtspunkten, keine speciellen Vorschriften für die Drainage angeben, theilt in einer neueren Arbeit Bode (18) eine besondere Methode mit.

Nach ausgiebiger Säuberung der Peritonealflächen durch reichliche Berieselung unter Anwendung des Eventrirens soll nahe der Radix mesenterii dureh dieses an einer gefässlosen Stelle ein starkes Drainrohr (Hauptdrain) zu beiden Seiten der Wirbelsäule rechts und. links über dem Colon durch besondere Hautschlitze nach aussen geführt werden. Ausser diesem wird je eins von den beiden Seitenöffnungen und ein viertes vom medialen Laparotomieschnitt in die Arehiv f. Gynäkologie. Bd, 63. H. 1 u. 2. 
tiefsten Punkte des kleinen Beckens gelegt, weitere je nach Nothwendigkeit in die Leber, Magen und Milzgegend.

Interessant ist seine Beobachtung, dass die Reposition der eventrirten Därme wegen der bei der Berieselung eintretenden Peristaltik leicht von Statten gehe. Auch hat sich trotz der Eventration bei den reichlichen Spülungen ein vorher kleiner frequenter Puls stets gehoben.

In den ersten Tagen nach der Operation werden nach seiner Methode zwei- bis dreimal täglich durch das Hauptdrain die Spülungen fortgesetzt, wodurch noeh nachträglich bedeutende Eitermengen entfernt wurden.

Auch die nachträglichen Spülungen haben, ohne Beschwerden zu machen, peristaltische Bewegungen ausgelöst, und so zum Wohlbefinden der Patientin viel beigetragen.

Wir sind mit diesen letzten Anführungen schon zum Theil auf das Capitel der Nachbehandlung übergegangen.

Sie muss vor allem eine stimulirende sein.

So lange noch Brechneigung besteht, darf per os nichts gereicht werden, es sind vielmehr in dieser Zeit möglichst reizlose Nährklystiere am Platze. Zur Anregung der Herzthätigkeit bezüglich zur Steigerung des Blutdrucks kommen Campher, Digitalis und Kochsalzinfusionen in Betracht.

Ein weiteres Augenmerk ist darauf zu richten, die noch zurückgebliebenen toxischen Substanzen möglichst bald aus dem Körper eliminiren zu helfen. Die Anhänger der Spülungen post operat. wollen dies dadurch mit erzielen, Michaux (1) und Monod (2) ihrerseits durch intravenöse Kochsalzinfusionen. Meist zeigt der Darminhalt solcher Kranken erhöhte Virulenz; für seine Entleerung Anregung zu schaffen, wird deshalb durchaus nothwendig sein. Aus unseren beiden Fällen ersehen wir, wie grade mit dem Einsetzen der ziemlich profusen diarrhoischen Stühle auch eine merkliche Besserung im subjectiven wie objectiven Befinden eingetreten ist.

Unsere Nachbülfe bestand in der $\Lambda$ pplication von Einläufen. F1oderus (24) empfiehlt Calomelbehandlung $0,05-0,1$ in Oblaten alle zwei Stunden bis $0,25 \cdots 0,4$ gegeben sind.

Mac Cosh(26) injicirt sogar sehon während der Operation, wie wir oben erwähnt haben, zur Anregung der Peristaltik in den incidirten Darm Magnesiasulfatlösung. 
Dass der weitere Heilungsverlauf zumeist ein protrahirter und vielfach complicirter ist, kann nicht weiter auffallen.

Vielfach finden wir die Beobachtung der Bauchdeckenabscesse.

Auch in unsern beiden Fällen ist ein solcher eingetreten und hat einen nicht unbedeutenden Bauchbruch hinterlassen.

Von der Vorsichtsmaassregel, bei eitrigen Processen in der Bauchhöhle die Bauchwunde zu übernähen, die sonst principiell

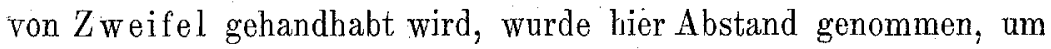
die Operation thunlichst zi beschleunigen.

Ziehen wir nun schliesslich das Facit aus unserer Betrachtung, so können wir etwa folgende Schlusssätze aufstellen:

Aussicht auf Heilung ist bisweilen noch in äusserst ungünstig erscheinenden Fällen von Peritonitis vorhanden. Die Operation ist deshalb auch hier als äusserstes Hilfsmittel zu versuchen.

Je frühzeitiger, desto besser ist vielfach die Prognose, die jedoch niemals, auch nicht annährend richtig gestellt werden kann. Der Eingriff ist thunlichst einfach zu gestalten. Er soll sich im wesentlichen auf die Entleerung und Drainage der Exsudatmassen beschränken.

$\mathrm{Ob}$ es besser ist, mit ausgiebigen Spülungen oder trocken aseptisch zu arbeiten, müssen weitere Erfahrungen lehren.

Nach der Operation ist eine möglichst stimulirende und roborirende Therapie am Platze.

\section{Literatur.}

1. Michaux, Traitement de la septicémie peritonéale par le sérum artificial et autres moyens connus. Bullet. et memoires de la Société de Chirurg. de Paris. 1896. No. 1, 2.

2. Monod, De l'hydrorrhé péritonéale. Dixième congrès de chirurgie. La semaine médicale. 1896. No. 53 .

3. Körte, Chirurg. Beh. d. allgem, eitrigen Bauchfellentzündung. Verh. der deatschen Ges. für Chir. XXI. S. $131 \mathrm{ff}$.'

4. Derselbe, Weiterer Bericht über die chirurg. Behand̄lung der diffusen Bauchfellentzündung und Discussion. Ebenda. XXVI. S. $15 \mathrm{ff}$. 
5. Abbé, Prognose u. Behandl. der allgem. Peritonitis. Centralbl. f. Gyn. XXI. 1232. Med. news. New York 1897. Mai.

6. von Erlach, Zur Behandlung der operativen Peritonitis. Wiener klin. Woch. 98. No. 13 .

7. Oberst, Ein Fall von Perforationsperitonitis. Centralblatt für Chirurgie. XII. S. -345 .

8. Laws on Tait, Discuss. on abdominal section. Edinb. med.journ. 1886. Mai, Jani.

9. von Winckel, Behandlung der von den weibliohen Genitalien ausgeh. Fintzündungen des Bauchfells ete. Jena. 1897.

9a. Derselbe, Ueber die chirurg. Beh, der von den weibl. Genital. ausgeh. Bauchfellentz: Sammlung klin. Vortr. N. F. 201.

10. Sippel, Zur Kenntniss der septischen Peritonitis. Centralblatt für Gyn. 1896. No. 12.

11. Senn, Classification und chir. Behandlung der acuten Peritonitis. Wien. med. Blätter. 1897. $38,39,40,43$.

12. Faure, A propos de quelques interventions d'urgence sur la cavité abdominale. Gazette des hopitaux. 1897.

13. Forgue, Traitement de péritonites aiguës. Gazette médicale. 1897. No. 48,49 .

14. Deanesley, The treatment of acute general peritonitis etc. British medical journal. 1898. Febr. 12.

15. Schloffer, Zur Therapie der acuten eitrigen Peritonitis. Prager medic. Wochenschi. 1898. No. 1 u. 5.

16. Vallas, Lyon med. 1899. Juli 30 .

17. Berard, Arch. prov. de chir. T. V. p. 235.

18. Bode, Eine neue Methode der Peritonealbehandlung und Drainage bei diffuser Peritonitis. Centralbl. für Chir. 1900. No. 2'.

19. W. Sykow, Soll man die Banchhöhle bei progressiv. Peritonit. waschen oder nicht? Chirurg. 98. Okt.

20. Gould, Twelve consecutive cases of acute general peritonitis. The Lancet 1898. Jan. 1.

21. Picherin et Petit, Infection péritonéale post-opératoire à staphyloeoques. La sémaine médicale. 1895 . p. 385.

22. Maxwell, The treatment of septic peritonitis by irrigation. The journal of Americ. Med. Ass. 1898. Sept. 10.

23. Floderus, Chirurgische Behandlung der Perfor. periton. Archiv für klin. Chir. 1897. S. 55.

24. Finney, Five succesful cases of general suppurative peritonitis etc. John Hopkins Hospital Bull. 97. Juli.

25. Mac Cosh, The treatment of general septic peritonitis. Annals of surgery. 1897. Juli, Aug.

26. Gluck, Vorschlag zur offenen Behandlung von Laparotomiewunden bei peritonealer Infection. Archiv für Kinderheilkunde. 1897. Bd. 23. Heft $1-3$.

27. Poncet, Traitement chirurgical des péritonites infectieuses généralisées post-opératoires. 'Lyon médical. 1897. No. 26. 
28. Jullien, Curabilité de la péritonite post-opératoire. Médicine moderne. 1891. No. 24.

29. Juillard, Péritonite purulente aiguë généralisée. Deux laparotomies. Guérison. Revue médicale de la Suisse romande. 1895. No. 10.

30. Noltschini, Beiträge zum Studium der secundären Laparotomie. Centralbl. für Gyn. XXI. S. 1162.

Siehe ausserdem die bei Körte und Winckel zusammengestellte Literatur. 